\title{
What's in a name? Comments on the use of the terms 'accident' and 'injury'
}

\author{
Polly E Bijur
}

The prevailing opinion in the public health community is that the use of the word 'accident' is detrimental to the efforts to describe, understand, analyze, and prevent the complex person-environmental interactions that lead to bodily injury. This is due to connotations of fate, chance, and unpredictability associated with the word, and even more so with the adjective, 'accidental'. As a result, the public health, pediatric, and medical literature over the past decade has increasingly replaced the word accident with the term 'injury', which is neutral with respect to causation, intentionality, and predictability. While this shift may have contributed to the increased scientific interest in the phenomenon, it has left substantial conceptual and linguistic problems in its wake.

Put quite simply, an injury is not an accident. To paraphrase Haddon, an injury results from transmission of energy from the environment to the human body when the quantity or nature of the energy is sufficient to cause damage to tissues. If the transmission of energy occurs over a relatively brief time, as in, for example, a fall from a height or a motor vehicle collision, it can be considered a discrete event that can be easily distinguished from the resulting injury or injuries. The major conceptual model used to assess etiology and identify targets for counter measures, the Haddon matrix, clearly distinguishes between the human-environmental event and the resulting injury. Haddon dropped the term accident in his later work; however, he insisted on addressing the personenvironment interchange in its full complexity, with the practical aim of identifying the phases of the process most amenable to intervention. Just as the single word, accident, did not adequately convey the complexity of the phenomenon, and in fact added a misleading etiologic assumption, the word, 'injury', only represents the outcome of a process in which an event, previously referred to as the accident, plays a central part.

Banishment of the word accident from the injury researcher's lexicon has resulted in some practical difficulties, in addition to the conceptual problem of conflating the event and the outcome. When referring to multiple injuries that occur from a single human-environmental interaction, some term needs to be substituted for the old ' $A$ ' word. When referring to events with injury producing potential that do not result in injury, some other term is needed. When referring to prevention of the events that lead to injury, we can list specific types of events (for example, motor vehicle crashes, fires, poisonings, assaults), but we have no term that encompasses them all.

In seeking a solution to the loss of the ' $A$ ' word we need to distinguish between scholarly usage and popular usage. The word 'accident' is deeply ingrained in the public mind and its use has remained untouched by the academic substitution of the word injury. Much as injury researchers may emphasize the continuum between intentional and unintentional injuries, the media and the public clearly make a distinction between injuries for whom no one is held directly responsible (accidents), and intentionally inflicted injuries. And, while decades of research have made it clear that there are seasonal, geographic, epidemic, and demographic variations in the occurrence of the events leading to injuries, these events are still perceived as largely random. Thus, those who work at the interface of academia and the public are likely to need to continue using the word 'accident', while hammering home the point that they are also preventable and predictable.

For the academic community, it would be advantageous to adopt a common term for the events that result in injury, or have the potential for injury. Given connotations of the word 'accident', the animosity to it over the past 10 to 20 years, and inclusion of clearly intentional injuries in the public health field of injury control, it is inadvisable to resurrect the term. One solution would be to use one of the multiword phrases that describe the phenomenon-'energy exchange'. 'personenvironment interaction', 'injury producing event'. These do not trip lightly off the tongue, nor do they lend themselves to word limits in abstracts. Another solution would be to find and agree to a neologism - a new word which telegraphically conveys the meaning of the phenomenon. Acronyms, such as IPE (for injury producing event) and PIPE (for potentially injury producing event) may serve the purpose. Although new terms are inherently unfamiliar and awkward, they have been successfully adopted in other fields, albeit characteristically in response to a technological development (for example, PET, CAT, EEG).

As for the name of this journal, it seems reasonable and appropriate to underscore its primary purpose. Prevention of events leading to injury may be an integral component of injury prevention, but ultimately it is the adverse impact of these events on the human body that is of paramount importance. 